\title{
Caratteristiche morfo-sintattiche e funzioni del discorso indiretto libero in italiano
}

\section{HASSNAA MAHMOUD AHMED MAHMOUD}

Docente assistente presso il dipartimento d'italiano

Facoltà Al-Alsun, Università di Minia

Relatore: prof. Sherif Abo Elmakarem

Professore associato presso il dipartimento d'italiano

Facoltà Al-Alsun, Università di Minia

\begin{abstract}
$\underline{\text { Abstract }}$
La tesina tratta principalmente le funzioni del discorso indiretto libero in italiano e le sue caratteristiche morfo-sintattiche. All'inizio, presenterò definizione del costrutto: è generalmente una forma ibrida fra discorso diretto e discorso indiretto.

Studierò, in primo luogo, le funzioni di questo tipo di discorso. Esaminerò, in secondo luogo, i segnali morfo-sintattici del discorso indiretto libero attraverso uno spoglio attinto da Gli Indifferenti di Alberto Moravia.
\end{abstract}

Parole chiavi: discorso riportato, discorso indiretto libero, discorso indiretto, tempi verbali.

\section{Introduzione}

Il discorso indiretto libero, d'ora in poi DIL, è fenomeno assai complesso per la duplice natura linguistico-stilistica; è stato scoperto e fatto oggetto di analisi agli inizi del XX secolo, in Francia, per merito di Charles Bally. Successivamente su di esso si sono soffermati anche alcuni studiosi italiani, soprattutto negli anni 1950-'60, che hanno rivolto la loro attenzione essenzialmente al lato stilistico del fenomeno. Bisogna precisare che una considerazione linguistico-grammaticale non è stata completamente trascurata in questi studi, ma essi non hanno potuto giovarsi delle conquiste compiute dalla linguistica italiana degli ultimi decenni, per merito degli 
studi sul parlato, le quali, certamente, sarebbero risultate proficue per una comprensione e interpretazione complessiva del DIL e della sua genesi. La presenza del DIL nella narrativa del XIX secolo, infatti, si connette strettamente alle nuove poetiche del realismo e, in particolare, alla loro esigenza di avvicinare la lingua letteraria a quella parlata.

Questa tesina si concentra principalmente su due punti: le funzioni del DIL in italiano e i suoi segnali morfo-sintattici. Prima di tutto, presenterò una definizione del costrutto: è una forma ibrida fra discorso diretto e discorso indiretto, è libero dai vincoli della dipendenza sintattica. Studierò, in primo luogo, le funzioni del DIL: esprimere i discorsi e qualche volta i pensieri dei personaggi, penetrare nell'intimo del protagonista per cogliere i sussulti della sua coscienza, essere strumento particolarmente adatto al discorso narrativo, consentire l'entrata pur se indiretta del personaggio determinando una sorta di fusione delle due voci (la polifonia) e usarlo come mezzo di distanziamento ironico dai personaggi. 


\section{Definizioni}

Questa forma è definibile come una costruzione ibrida tra quella del DD e quella del DI. Si tratta, quindi, di una forma la cui definizione e la cui descrizione sono molto controverse. Nel DIL si può fare a meno di «una cornice esplicita», non vi è necessariamente né verbo di dire né subordinatore e le forme dei pronomi personali sono equivalenti a quelle che si avrebbero nel DI; talvolta questa equivalenza è riscontrabile anche per il tempo verbale e gli avverbi spaziali e temporali. Il DIL, come il DD, può presentare «enunciati non dichiarativi e costruzioni marcate (inversioni, dislocazioni, frasi incomplete, ecc.) ed elementi di tipo espressivo (vocativi, esclamazioni, ecc.)» ${ }^{1}$. Nella lingua scritta letteraria, aggiunge Calaresu ${ }^{2}$, il DIL è talvolta indicato con il corsivo; nella lingua orale, invece, è segnalato spesso da un'intonazione, o da un colore di voce particolare. A causa del suo supposto carattere letterario, viene spesso esemplificato nei manuali con esempi tratti da romanzi e racconti. Così come nel caso della definizione seguente di DIL, estratta da Dardano e Trifone $^{3}$ citati in Calaresu ${ }^{4}$. Si segnala in grassetto la parte corrispondente a un DIL:

«DIL: Nozione utilizzata soprattutto nell'analisi dei testi narrativi moderni per indicare che il discorso del personaggio è riportato dall'autore in forma indiretta, mantenendo però alcuni caratteri tipici della forma diretta. Si veda p. es., il seguente esempio dell'Esclusa di Pirandello: - Oh Dio, Paolo, che t'è successo?

- Niente. In una stanza della conceria, al buio, qualcuno (e forse a bella posta!) s'era dimenticato di rinchiudere la ... come si chiama? Sì ... la caditoia, ecco, sull'assito, ed egli, passando patapumfete! Giù» ${ }^{5}$.

La risposta del personaggio è riportata in forma indiretta, ma confermando moduli propri del parlato. Si può dire, quindi, che il DIL costituisce una struttura alternativa rispetto al DD o DI ed ha principalmente lo scopo di vivacizzare lo stile.

\section{Funzioni del Discorso indiretto libero}

Tanto è vero che la netta maggioranza degli studi sul DIL abbiano come oggetto la prosa letteraria, mentre mancano - perlomeno in italiano - ricerche approfondite sulle funzioni del DIL nella scrittura funzionale e nella comunicazione orale. Nella prosa letteraria, afferma Mortara Garavelli ${ }^{6}$, tradizionalmente si considera che il DIL nasca dall'esigenza di avvicinare la lingua letteraria alla lingua viva. Da un punto di vista sintattico il DIL è visto come un DI alleggerito dagli elementi grammaticali più fastidiosi (verba dicendi, che subordinativo, ecc.).

Infatti, Il DIL serve per esprimere i discorsi e qualche volta i pensieri dei personaggi; in tal caso i pensieri del narratore e quelli dei protagonisti confluiscono, e diventa molto difficile distinguere tra narratore e personaggio: il DIL diventa allora strumento per vedere cose e fatti con gli occhi e le parole dei personaggi: 
- Difficile impegno; Carla doveva avere dei principi o forse, chissà? Amare qualchedun altro; difficilissimo... [...] di queste due maniere quale la migliore?... La seconda, indubbiamente... molto più facile lasciarle capire certe cose, che dirle... e poi, [...]. (A. Moravia, Gli Indifferenti, p. 243)

Inoltre, il DIL non è tanto strumento espressivo, quanto di scavo e di analisi, per portare a galla impressioni e pensieri sovente gelosamente custoditi: rivela il dramma dell'oscura vita quotidiana ${ }^{7}$ :

- Michele fece ancora un passo avanti: "buffonello?" era o non era una grave ingiuria ledente il suo onore e la sua reputazione questo "buffonello"? A giudicare dalla propria indifferente calma, no; se invece si pensava al significato della parola e al poco amichevole sentimento che l'aveva ispirata, si certamente. "Agire" egli pensò con una specie di ebbrezza; "per es. schiaffeggiarlo". Non c'era un minuto da perdere, Leo era là, a un passo di distanza, appoggiato nel vano della finestra, contro la tenda di velluto, quella guancia che egli doveva colpire era in piena luce, larga, sanguigna, ben nutrita, ben rasata, tutta la mano ci sarebbe entrata, non c'era da temere di non cogliere nel segno... dunque... (A. Moravia, Gli Indifferenti, pp. 69- 70).

Dunque, al DIL è affidata la possibilità di penetrare nell'intimo del protagonista, di cogliere i sussulti della sua coscienza: nell'esempio, si susseguono le varie considerazioni del protagonista riguardanti un insulto da parte di Leo; segnano una progressiva rabbia nella sua interiorità, in modo tale da farlo pensare a colpirlo.

- Quel che più la irritava era l'idea che l'amica potesse crederla infetta di dabbenaggine d'ingenuità; dalla rabbia mentre camminava faceva gli occhi piccoli e stringeva i denti, non si era mai sentita così spietata: se Lisa fosse stata morente per la sete le avrebbe rifiutato l'ultimo bicchier d'acqua, se affamata l'ultimo boccone; se poi l'amica fosse diventata improvvisamente povera, avrebbe potuto supplicarla in ginocchio e baciarle le mani, ella non le avrebbe dato neppure un centesimo di roba; nulla; [...]. (A. Moravia, Gli Indifferenti, p. 208)

Il DIL svolge in questo contesto un ruolo preciso: esso non è tanto generica espressione dei pensieri del protagonista che vengono spesso riportati anche in forma diretta, ma sempre «segno dell'improvviso affiorare di una tensione interiore, di un conflitto angoscioso» ${ }^{8}$.

Quanto detto connota il DIL, come strumento particolarmente adatto al discorso narrativo, giacché esso consente di realizzare una forma di polifonia basata sulla «simultaneità»: non una voce, quella del narratore o quella del personaggio, bensì tutte e due le voci. È attraverso il DIL, quindi, che «il narratore consente l'entrata pur se indiretta del personaggio determinando una sorta di fusione delle due voci (la polifonia)» ${ }^{9}$. Perciò, il DIL è potuto apparire quale «luogo privilegiato per lo studio del punto di vista, e della plurivocità come fattore di una nuova dimensione 
linguistica» ${ }^{10}$. L'inevitabile conseguenza è che il DIL riflette i suoi effetti sulla ricezione del messaggio, inducendo una percezione diversa rispetto a quella del DD (in cui il personaggio comunica direttamente, e si ha la forza massima dell'io che produce il messaggio) e del DI (in cui la forza dell'io produttore del messaggio raggiunge il suo livello minimo).

Infine, Mortara Garavelli ${ }^{11}$ aggiunge alle funzioni che sono state attribuite al DIL, l'usarlo come mezzo di distanziamento ironico dai personaggi o all'opposto di identificazione e di empatia con questi, veicolo del cosiddetto 'flusso di coscienza'.

\section{Segnali morfosintattici}

Sotto questo punto incontreremo gli indicatori primari di DIL, cioè le strutture portanti del DIL:

\subsection{Sistema dei tempi verbali}

\subsubsection{Il presente}

A parere di Cane, l'uso del presente assume un particolare valore all'interno della narrazione in generale, e all'interno del DIL in modo speciale. Infatti, si usa il presente nel DIL «quando si tratta di un'affermazione generica, o di valore universale» $^{12}$ :

- Si sentiva leggera e turbata, come di chi è in procinto di partire e siede per l'ultima volta alla tavola famigliare, mangiando in fretta e pensando all'imminente viaggio... invece la madre le appariva ferma per sempre al suo posto, pianificata in quell'atteggiamento... (A. Moravia, Gli Indifferenti, p.219)

Nell'esempio precedente, si osservino il presente (è, siede) che esprimono un fatto generale e quindi hanno un valore generico.

D'altra parte, talora quando si vogliono esprimere vivaci sentimenti o eventi inaspettati, il presente subentra al passato. Non va dimenticato che il DIL, in tutti i casi, significa «tensione e una specie di eccitamento, di vario grado naturalmente» ${ }^{13}$ :

- [...] e si capiva che ella, sedutagli al lato, gli si era accasciata sulle ginocchia, aggrappandosi con la mano a quelle spalle, come chi vuole supplicare e non osa guardare in faccia. (A. Moravia, Gli Indifferenti, p. 123)

- Anche qui era evidente una leggera esagerazione, ma Michele non la rilevò; tanto a che cosa poteva servire? A certe cose non si può metter rimedio. (A. Moravia, Gli Indifferenti, p. 221)

- Quelle lacrime colate sul volto imbellettato, versate in quel momento,

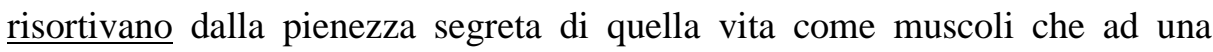
leggera contrazione affiorano improvvisamente sotto la pelle. Quell'anima era 
intera, coi suoi vizi e le sue virtù, e partecipava delle qualità di tutte le cose vere e solide [...]. (A. Moravia, Gli Indifferenti, p. 248)

Nei primi due esempi, Moravia passa dall'imperfetto al presente, ma non per dare valore universale a ciò che pensa il protagonista, o non per esprimere un pensiero staccato dalle contingenze temporali. Si tratta, in realtà, di un vero e proprio cambiamento di persona, come se nei pensieri della protagonista si inserisse il commento dell'autore, che non contento d'indagare continuamente l'animo dei suoi personaggi, entra anche direttamente in essi, per fermarsi a contemplare il mistero che è in ognuno. Analizzando attentamente questi esempi appena citati, ci si accorge che si tratta di un DIL, se non da un punto di vista grammaticale, almeno da quello stilistico: la scelta di certi particolari (A certe cose non si può metter rimedio, Quell'anima era intera, coi suoi vizi e le sue virtù, ecc.) pare fatta dalla commozione di colui che guarda. Insomma, ci troviamo di fronte ad un caso estremo di identificazione tra l'autore e i suoi personaggi.

Il cambiamento del tempo con l'apparizione del presente segna l'acme della storia. Questo presente, inserito nel DIL «corrisponde, sotto un certo aspetto, al presente storico, usato normalmente ad esprimere affetti e stati d'animo di una determinata carica emotiva» ${ }^{14}$.

Inoltre, ci si serve del presente per introdurre constatazioni su cose e persone, e soprattutto descrizioni di paesaggi, che quasi si staccano dal resto della narrazione:

- Così atteggiata, vestita elegantemente, eccola entrare ${ }^{15}$ nella sala affollata di un albergo; suo marito la segue, Leo, un po' più calvo, un po' più grasso, ma non molto cambiato; $\underline{\text { si seggono, }}$ prendono il tè, ballano, molti la guardano $\mathrm{e}$ pensano: "bella donna bella ma cattiva..." [...] tutti mormorano, pensano, la guardano; ella sta seduta accanto a quel suo marito, tiene le ginocchia accavalciate, fuma... effetto di gambe, il vestito è succinto, la scollatura è profonda... tutti l'osservano con bramosia come se volessero morderla; ella risponde loro con sguardi pieni d'indifferenza... Una camera... ecco: la signora Merumeci, in ritardo per qualche visita di obbligo, corre incontro al suo amante; tra quelle braccia perde quella sua durezza di statua, queste donne rigide sono sempre le più ardenti, ridiventa fanciulla, piange, ride, balbetta, è come una prigioniera liberata che rivede alfine la luce... la sua gioia è bianca, tutta la stanza è bianca, ella è senza macchia tra le braccia dell'amante... la purezza è ritrovata. Poi, quando vien l'ora, stanca e felice, torna alla casa coniugale e ricompone sul suo volto l'abituale freddezza... la sua vita continua così per degli anni... molti la invidiano... ella è ricca, si diverte, viaggia, $\underline{\text { ha } u n ~ a m a n t e, ~ c h e ~}$ più? Tutto quel che può avere una donna lo ha... (A. Moravia, Gli Indifferenti, pp. 311-312)

Normalmente le descrizioni vengono fatte dal narratore, ma in questo esempio sono descrizioni in DIL: è da notare, l'uso del tempo presente, che dà chiaramente a tutto 
l'esempio il tono di un normale commento, e di cui l'autore si assume tutta la responsabilità. Altri elementi classici, affettivi e grammaticali del DIL si individuano agevolmente, via l'uso dell'infinito (entrare), l'interrogazione (che più?), la frase nominale (la signora Merumeci, in ritardo per qualche visita di obbligo), la trasposizione dei pronomi e delle voci verbali dalla prima persona alla terza.

Altre volte, il presente si utilizza per fissare l'attenzione del lettore sui fatti essenziali della vicenda:

- L'importanza di questa sua visita era per lui evidente ed estrema: era l'ultima prova della sua sincerità, dopo il fallimento della quale o egli sarebbe restato in queste provvisorie condizioni di dubbio e di ricerca, o si sarebbe incamminato per la via opposta, quella di tutti, dove le azioni non sono sorrette da alcuna fede o sincerità, si valgono tutte tra di loro e si accumulano in belle stratificazioni sullo spirito dimenticato fino a soffocarlo; ma se la prova riusciva, tutto sarebbe mutato: avrebbe ritrovato la sua realtà concreta come un artista ritrova l'ispirazione dei tempi più felici; una nuova vita sarebbe cominciata, la vera, la sola vita possibile. (A. Moravia, Gli Indifferenti, $\mathrm{p}$. 240)

- Ella non si vede, sta supina, ma da quegli sguardi dell'uomo capisce di aver le membra sparse di Dio sa che macchie o segni, di esser mutata anche per lui, straniero, da quella Carla che era prima della tresca; restano così ambedue, $\underline{\text { si }}$ guardano, non si muovono ... (A. Moravia, Gli Indifferenti, p.184)

Gli esempi sopracitati si devono attribuire al personaggio, il quale rivive mentalmente l'episodio. E solo lui stesso che è capace di scegliere precisamente alcuni aggettivi come (l'ultima prova, nuova vita, la vera, la sola vita possibile), di valutare la situazione (queste provvisorie condizioni di dubbio e di ricerca), come nel primo esempio; mentre nel secondo cita qualche espressione religiosa di uso popolare (aver le membra sparse di Dio sa che macchie o segni), il che è considerato uno dei più importanti indizi di DIL.

Inoltre, il presente si riferisce a fatti reali o realizzabili:

- Il secondo; Lisa si rovinava, faceva dei debiti, impoveriva, diventava cenciosa e mendicante; tutti l'abbandonavano; oppure dopo una grave malattia restava smagrita, imbruttita, grigia e, chissà, son cose che avvengono, forse ebete e mentecatta, forse cieca... un viso scarnito, degli occhi bianchi, una fronte incerta che urta contro i mobili e contro le persone... il dito di Dio, la punizione del Cielo, son cose che succedono... (A. Moravia, Gli Indifferenti, p. 208)

- Capiva che bisognava una buona volta vincere la propria indifferenza e agire; senza alcun dubbio l'azione gli veniva suggerita da una logica estranea alla sincerità; amor filiale, odio contro l'amante di sua madre, affetto famigliare, tutti questi erano sentimenti che egli non conosceva... ma che importava? Quando non si è sinceri bisogna fingere, a forza di fingere si finisce per credere; questo è il principio di ogni fede. (A. Moravia, Gli Indifferenti, p. 210) 
Nel primo esempio, le due frasi (son cose che avvengono, son cose che succedono) esprimono che si tratta di eventi normali o possono essere realizzate; e nel secondo, il presente in (Quando non si è sinceri bisogna fingere, a forza di fingere si finisce per credere; questo è il principio di ogni fede) è molto espressivo a chiarire il significato inteso. Allora, si nota attraverso gli esempi fin qui citati che il presente rompe il monotono succedersi degli imperfetti.

Per concludere, possiamo concordarci con il parere di Herczeg ${ }^{16}$ che afferma che il DIL è, dal punto di vista grammaticale, «un insieme di fenomeni devianti dalla sintassi regolare, quale è codificata dalle grammatiche». Il DIL rispecchia il parlar vivo, ma contemporaneamente è costretto a ubbidire a determinate regole severe del DI; dunque, la sintassi del DIL rispecchia questa duplicità.

\subsubsection{Il condizionale}

In quanto segue, esponiamo alcuni esempi di DIL tratti da Gli Indifferenti in cui è usato il condizionale composto, di valore citativo nei primi due esempi:

- [...] quel sorriso era ingiurioso; un uomo forte, un uomo normale se ne sarebbe offeso e avrebbe protestato; lui invece no ... lui con un certo avvilente senso di superiorità e di compassionevole disprezzo restava indifferente ... ma volle per la seconda andar contro la propria sincerità «protestare», pensò «ingiuriarlo daccapo». (A. Moravia, Gli Indifferenti, p. 33)

Si osservano i due condizionali (se ne sarebbe offeso e avrebbe protestato) di uso citativo nell'assenza di una cornice e il subordinante $c h e$. Che si tratti di DIL, è chiaro da vari indizi: l'uso della frase nominale (un uomo forte, un uomo normale), la locuzione (lui invece no) con i punti sospensivi propri del DD; subito dopo s'interrompe il DIL con la narrazione dell'autore.

- Ella fece di nuovo il vano gesto di respingerlo, ma ancor più fiaccamente di prima, ché ora la vinceva una specie di volontà rassegnata: perché rifiutare Leo? Questa virtù l'avrebbe rigettata in braccio alla noia e al meschino disgusto delle abitudini; e le pareva inoltre, per un gusto fatalistico di simmetrie morali, che questa avventura quasi famigliare fosse il solo epilogo che la sua vita meritasse; dopo, tutto sarebbe stato nuovo; la vita e lei stessa; guardava quella faccia dell'uomo, là, tesa verso la sua: "Finirla", pensava "rovinare tutto..." e le girava la testa come a chi si prepara a gettarsi a capofitto nel vuoto. (A. Moravia, Gli Indifferenti, p. 8)

Il costrutto è altrettanto regolare da un punto di vista formale; anche qui si osserva il condizionale composto citativo, ma, accanto a questi, ha notevole sviluppo la frase interrogativa, altro mezzo assai usato dall'autore all'interno del DIL per indicare l'angosciosa indagine di Carla sulla realtà e soprattutto sul perché di rifiutare il matrimonio con Leo. Inoltre, si rivela critico, secondo quanto osserva Franca 
Schettino, l'aggettivo del gruppo «meschino disgusto» ${ }^{17}$ riferito a Carla. Con la qualificazione del sostantivo disgusto giunge al lettore anche un giudizio, un commento allusivo di tinta ironica, d'origine tonale, riguardante, quindi, il ritmo e la struttura della frase. Leggendo oltre nello stesso esempio, si nota, ancora secondo Schettino $^{18}$, che la contaminazione ironica è radicata nel costrutto sintattico e precisamente nel DIL impiegato ad illustrare lo stato d'animo del protagonista (Carla). L'impressione totale sul lettore è di una descrizione caricaturale come se il narratore, invece di narrare oggettivamente lo stato d'animo del personaggio, lo avesse impersonato mimicamente con allusioni e motteggi.

- Questa immaginazione la colpì a tal punto che restò ferma davanti allo specchio, guardandosi; ecco le pareva di vedersi, seduta sulle ginocchia di Leo, in atto di dargli un colpetto sulla guancia, o di appoggiargli affettuosamente la testa contro il petto, domandandogli sottovoce il denaro per qualche suo vestitino; oppure sarebbe andata insieme con l'amante da quella celebre modista, avrebbe ordinato tre o quattro di quei cappelli di Parigi, novità della stagione, che tanto le piacevano; (A. Moravia, Gli Indifferenti, p. 115)

In questo esempio, è detto esplicitamente che si tratta di immaginazione, quindi di un pensiero. Si nota l'uso del DIL attraverso alcuni noti criteri e soprattutto dall'uso di: l'imperfetto, il condizionale, ecco, pronomi trasposti, avverbi affettivi (affettuosamente, sottovoce); e parole esprimenti l'imprecisione (tre o quattro) ${ }^{19}$ che è un altro indizio di incertezza.

- E già immaginava come tutto questo sarebbe avvenuto; ecco... egli l'avrebbe riattirata al suo fianco, e guancia contro guancia, le avrebbe mormorato in orecchio le dolci parole, già al solo pensiero se ne sentiva tutta commossa e quasi consolata, quando, repentinamente, una terribile paura l'agghiacciò. (A. Moravia, Gli Indifferenti, p. 187)

Qui, il DIL si deve attribuire alla protagonista (Carla) la quale rivive mentalmente un evento. La presenza del personaggio si sente inoltre nella descrizione precisa (le dolci parole, tutta commossa, quasi consolata), e nella scelta di alcune forme discorsive (già).

- “Avanti, avanti” pensò, e oscuramente si meravigliava di questa sua capacità di scoprire sempre nuove abiezioni; quando ne sarebbe arrivata la fine? "Bisogna arrivare fino in fondo". Sorrise pallidamente... dunque se Leo non avesse voluto sposarsi... anche quest'ipotesi era probabile... in tal caso un altro accordo avrebbe potuto farsi tra le due parti contraenti... Leo avrebbe dato $i$ soliti quattrini e in considerazione della giovinezza intatta, della bellezza di Carla, gli avrebbe stata richiesta una somma due, tre volte maggiore di quella che sarebbe bastata per Lisa matura e corrotta... ad ogni merce il suo prezzo... ed egli... ed egli in cambio si sarebbe impegnato, sicuro, in tale atmosfera [...] si sarebbe impegnato a facilitare le cose presso la sorella. Difficile impegno; Carla doveva avere dei principi o forse, chissà? Amare qualchedun altro; difficilissimo... [...] 
di queste due maniere quale la migliore? ... la seconda, indubbiamente... molto più facile lasciarle capire certe cose, che dirle... e poi, [...]. (A. Moravia, Gli Indifferenti, p. 243)

La seconda parte del periodo che comincia da (dunque se Leo non avesse voluto sposarsi... anche quest'ipotesi era probabile...) appare palesemente sciolta dal collegamento con una qualsivoglia frase introduttiva citante: manca un verbo di dire, mentre si avverte quella discordanza improvvisa, discordanza che determina un mutamento rispetto alla frase precedente, e produce uno stile che non può dirsi né diretto (per l'uso della terza persona) né chiaramente indiretto, (per la presenza dell'interrogazione di queste due maniere quale la migliore?...) che apporta un'enfasi tipica del parlato, e dunque incompatibile con il discorso indiretto nel quale tali marche soggettive non sono utilizzabili. Infatti, l'esempio è abbondante di altri indizi di DIL: forme discorsive (dunque, in tal caso, due o tre volte), frase nominale (ad ogni merce il suo prezzo), la locuzione che esprime incertezza (chissà?), e l'uso frequente dei punti sospensivi.

\subsubsection{L'imperfetto}

Dal punto di vista di Pasolini, il frequentissimo uso dell'imperfetto implica uno scrittore - narrante che, per un bisogno di "intercomunicabilità" col suo personaggio e un bisogno di espressività, «crea la condizione stilistica necessaria per rendersi narrante attraverso il suo personaggio $»^{20}$ : soprattutto nelle considerazioni amare o gioiose su condizioni presenti o passate: il pensare meditativo, il brontolare, ecc. Ma ci sono dei casi, in cui lo scrittore rinuncia fin da principio a essere scrittorenarrante, e s'immerge subito nel suo personaggio narrando tutto attraverso lui. Facendo così, uno scrittore intende «rendere fittiziamente oggettivo ciò che egli vuol dire» ${ }^{21}$. E ciò si vede chiaro negli esempi seguenti:

- Così gli era possibile di veder in Carla una fanciulla disonesta, nella madre una signora stupida e ridicola, in Lisa una donnaccia; per non parlare di Leo che di ora in ora, attraverso $i$ discorsi degli altri e le proprie troppo obbiettive impressioni, cambiava completamente, così, che se in un primo momento credeva di odiarlo, poco tempo dopo lo amava teneramente. (A. Moravia, Gli Indifferenti, p. 249)

Con l'imperfetto, autore e protagonista s'immergono al grado di farci sentire che è Michele stesso che esprime i suoi pensieri. Indizi chiari di DIL nel brano sono l'introduttore anaforico (così) che collega il DIL con quello che precede, e il notevole uso dell'ellissi - uso proprio del DIL - del verbo vedere in (nella madre una signora stupida e ridicola, in Lisa una donnaccia).

- La sua solitudine, le conversazioni con Lisa gli avevano messo in corpo un gran bisogno di compagnia e di amore, una speranza estrema di trovare tra tutta la gente del mondo una donna da poter amare sinceramente, senza ironie e senza 
rassegnazione: "Una donna vera” pensò; “una donna pura, né falsa, né stupida, né corrotta... trovarla... questo sì che rimetterebbe a posto ogni cosa". Per ora non la trovava, non sapeva neppure dove cercarla, ma ne aveva in mente l'immagine, tra l'ideale e materiale che si confondeva con le altre figure di quel fantastico mondo istintivo e sincero dove egli avrebbe voluto vivere; la musica lo avrebbe aiutato a ricostruire quest'immagine amata... ed ecco difatti, più che per la sua esaltazione e per il suo desiderio che in grazia della musica stessa, fin dalle prime note, formarsi tra lui e Carla quella immagine... era una fanciulla, lo indovinava dalla snellezza del corpo, dagli occhi, da tutto il portamento, assai graziosa in verità, quasi gli voltava le spalle e l'osservava attentamente, senza lusinga, senza ombra di lascivia, oh, no, avrebbe potuto giurarlo, ma con quella curiosità franca e attonita con la quale $i$ bambini guardano i loro coetanei: "la mia compagna", egli pensò: e già dei gesti, una specie di abbraccio, un sorriso, una mossa della mano, degli avvenimenti, passeggiate, conversazioni, si formavano e passavano nel cielo desideroso della sua fantasia, quando un chiacchiericcio fitto e sommesso ruppe l'illusione e lo ricondusse alla realtà. (A. Moravia, Gli Indifferenti, p.150)

Questo sentimento dominante di Michele, nell'esempio sopracitato, è espresso attraverso il DIL. È ovvio il contenuto soggettivo e sentimentale, ma tale contenuto proviene anche dall'uso di: qualche forma discorsiva (neppure, quasi, difatti), qualche aggettivo (istintivo e sincero, tra l'ideale e materiale) e l'interiezione (oh no).

Se volessimo trasformare l'imperfetto con il passato remoto, il tono del racconto diventerebbe molto più obiettivo, più distaccato: scomparirebbe il DIL, scomparirebbe cioè la compenetrazione tra personaggio e scrittore, essenza fondamentale per il DIL. Con l'imperfetto, spiega Herczeg ${ }^{22}$, è il personaggio che espone le scenette, le rivive, le sente, offrendogli un profondo senso di umanità. Invece, con il passato remoto, tutto si concentrerebbe intorno allo scrittore che espone una sequela di eventi, a cui egli non partecipa umanamente:

- La fanciulla alzò gli occhi: questo spirito gioviale e falsamente bonario inaspriva la sua impazienza: ecco, ella sedeva alla tavola famigliare, come tante altre sere; c'erano $i$ soliti discorsi, le solite cose, più forti del tempo, e soprattutto la solita luce senza illusioni e senza speranze, particolarmente abitudinaria, consumata dall'uso come la stoffa di un vestito e tanto inseparabile dalle loro face, che qualche volta accendendola bruscamente sulla tavola vuota ella aveva avuto la netta impressione di vedere i loro quattro volti, della madre, del fratello, di Leo $e$ di se stessa, là, sospesi in quel meschino alone; [...] (A. Moravia, Gli Indifferenti, op. cit., p.18)

Qui è usato l'imperfetto per esprimere ciò che pensava Carla in quel momento, cioè il proprio disgusto della solita vita con tutti i suoi noiosi dettagli.

- Ella (Lisa) allargò la tendina; $i$ vetri erano tutti imperlati di vapore, doveva far freddo; attraverso questa rugiada s’indovinavano dei colori vaghi, tenui e puri, 
un bianco, un verde come dissolti in un lago d'acqua; ella sguarciò con la mano questo vel liquido e vide subito un pezzo di tetto rossiccio di un aspetto così poco luminoso, così indifferente e opaco che non ebbe bisogno di guardar più in su per vedere se il cielo era grigio; si distaccò, fece macchinalmente qualche passo per la stanza ingombra. (A. Moravia, Gli Indifferenti, p.47)

Nell'esempio precedente, è facile delimitare il DIL: si trova tra due verbi al passato remoto (allargò, sguarciò), e il DIL consiste in una breve descrizione di una scenetta dopo la pioggia; una descrizione molto minuziosa a farci sentire che Lisa stessa che descrive.

Allora, non si tratta di un alternarsi degli imperfetti e dei passati remoti, ma dello scambio delle persone che riferiscono: quando si mette a parlare lo scrittore, emerge il passato remoto, mentre quando si tratta di un dialogo interiore, il contenuto messo al DIL deve avere contatti stretti con il protagonista. L'imperfetto, asserisce Mortara Garavelli ${ }^{23}$, per la sua indeterminatezza temporale si adatta all'ambiguità del DIL. Spiegando l'idea dell'indeterminatezza dell'imperfetto, Carla Bazzanella ${ }^{24}$ afferma che l'imperfetto si caratterizza per la maniera indeterminata con cui il processo verbale viene presentato. Dunque, l'imperfetto è imperfettivo perché esprime l'indeterminato. Inoltre, si crede di poter affermare che l'indeterminatezza con cui l'imperfetto presenta l'azione, la separa in qualche modo dal tempo reale in cui si svolge.

- Andò dritta alla porta della sua camera, in fondo al corridoio oscuro, entrò; subito la colpì l'aspetto intimo e caldo della stanza: ogni cosa era al suo posto, la lampada dal paralume rosa era accesa, la camicia di velo cilestrino stava distesa sul letto, le lenzuola erano piegate e aperte, tutto invitava al sonno: non c'era che da spogliarsi, cacciarsi sotto le coltri e dormire. (A. Moravia, Gli Indifferenti, p.162)

- Gli occhi le si empirono di lacrime; tutti erano colpevoli e nessuno, ma ella era stanca di esaminare se stessa e gli altri; non voleva perdonare, non voleva condannare, la vita era quel che era, meglio accettarla, che giudicarla, che la lasciassero in pace. (A. Moravia, Gli Indifferenti, p. 310)

Nei due esempi precedenti, c'è una grande scarsità di elementi formali, ma la descrizione è fatta dall'occhio del protagonista; possiamo dire che c'è un'immedesimazione ${ }^{25}$ tra il punto di vista del narratore e quello del personaggio. Inoltre, nel secondo, si riscontra una sequela di sintagmi staccati che danno un respiro diverso al DIL.

Per concludere, l'alternanza nell'uso dei tempi ${ }^{26}$ passati nella narrazione dipende dalle diverse caratteristiche aspettuali: l'imperfetto è un tempo imperfettivo 
ed ha la funzione di esprimere un evento nel corso del suo svolgimento o che dura per un certo arco di tempo, quindi si adatta bene a rappresentare i processi che fanno da sfondo all'azione narrativa vera e propria. Il passato remoto e il passato prossimo sono, invece, tempi perfettivi, cioè rappresentano un evento compiuto che ha avuto un inizio e un termine nel passato e «sono pertanto adatti a far procedere la narrazione». Per questo motivo nei racconti si osserva che spesso le descrizioni, che consistono in «sequenze statiche», sono affidate all'imperfetto, mentre la narrazione, che richiede dinamicità, è affidata ai due tempi perfettivi.

\subsubsection{L'infinito}

Gli infiniti, in alcuni casi, si armonizzano con le proposizioni circostanti in modo che i soggetti siano identici:

- Aveva dimenticato di distruggere o di portare con sé i pezzi dello scritto stracciato. Risalire a prenderli? (Fogazzaro, Leila: p.199) ${ }^{27}$

- [...] e ogni tanto tendeva una mano esitante e tastare lo spazio immenso e freddo che avanzava dietro, quella Siberia di tela, disabitata e ostile; era una sensazione sgradevole: come camminare per una strada buia sapendo di avere qualcheduno alle spalle. (A. Moravia, Gli Indifferenti, p.181)

- [...] Andremo a portare questa busta alla Montanina ... andare alla Montanina? (G. Verga, Mastro-don Gesualdo, p. 270) ${ }^{28}$

Negli esempi, il legame del sintagma infinitivale con la proposizione precedente è evidente: chi dimentica di portare con sé qualche cosa, può risalire, se vuole, a prenderla; e nell'altro ha teso la mano per tastare lo spazio. Nel terzo, il legame appare ancor più evidente, grazie alle parole che sono identiche; l'infinito riprende il verbo della proposizione precedente.

- Quel secondo sguardo gli bastava; evidentemente Lisa dubitava della sincerità del suo sdegno; come fare per convincerla? (A. Moravia, Gli Indifferenti, p. 267)

In questo esempio, la concatenazione logica, benché non così lampante come negli esempi precedenti, è pure evidente.

In alcuni casi di DIL vi sono infiniti di maggiore indipendenza: questi infiniti non si accordano con le proposizioni contigue, e talvolta anche i soggetti sono divergenti. Infatti, gli infiniti di questo tipo adducono elementi nuovi al discorso:

- Ella fece di nuovo il vano gesto di respingerlo, ma ancor più fiaccamente di prima, ché ora la vinceva una specie di volontà rassegnata: perché rifiutare Leo? (A. Moravia, Gli Indifferenti, p.8)

Il soggetto dell'infinito (rifiutare) non ha appigli facilmente riconoscibili nelle proposizioni precedenti, i quali non sono né conseguenza, né continuazione, neppure 
ripetizione delle frasi precedenti, ma costituiscono novità di fronte alle premesse. Così contengono il monologo interiore di Carla che si chiede perché rifiuta Leo.

Infatti, gli infiniti indipendenti hanno assunto tanto rilievo nel DIL, nel quale si condensano stati emotivi diversi: dubbio, inclinazione, stupore, indignazione, come si vede rispettivamente negli esempi seguenti:

- Ma la sua fantasia oppressa rifiutava il sarcasmo e dalla carezza di quelle dita traeva un pretesto per quelle appassionate immaginazioni: cercare Lisa in quella notte, stringerla finalmente al suo petto in un bacio sincero, definitivo... per un istante egli lottò contro questa sua debolezza. (A. Moravia, Gli Indifferenti, p.130)

- Ora eccola, pronta ad uscire, col cappellino ben calzato sugli occhi, la faccia incipriata, fresca, rosea, le labbra dipinte, due riccioli aguzzi sulle guance; eccola lasciar quello specchio appannato, quell'aria torbida, quella parete, quella sedia, e venirgli incontro. (A. Moravia, Gli Indifferenti, p.300)

- Separarmi da voi?

- Io farmi moglie di un chiozzotto come quello?29

Negli esempi citati, si nota un miscuglio di battute affettive che non contengono molto di speciale da comunicare; sono però di alta tensione, perché esprimono i sentimenti e gli atteggiamenti del protagonista, e anche dello scrittore di fronte ai vari avvenimenti.

\subsection{Indipendenza del DIL dal verbum dicendi}

Perché si attui questa essenziale condizione formale del DIL, il verbo introduttore o è espresso o è assente; nel primo caso è a reggenza indiretta (cioè, se transitivo, è a regime diretto sostantivo o in forma incidentale, altrimenti è intransitivo). Secondo Bendoni ${ }^{30}$, la conseguenza dell'impossibilità per siffatti verba dicendi di subordinare a sé il brano enunciativo, è l'assenza di quei «mots grammaticaux» che introducono il DI, cioè delle congiunzioni subordinative che, se, $d i$ : è per questo che si parla di DIL. In quanto segue, verranno esposti i casi in cui il verbum dicendi è espresso.

\subsubsection{I casi di un verbum dicendi espresso}

Ora, esporremo nei punti seguenti i casi in cui il verbum dicendi è espresso:

A. Quando il verbum dicendi precede il DIL, si tratta allora di un verbo intransitivo: - (Lo zio Crocifisso Piedipapera) parlavano a voce alta nelle stanze vuote, dove le parole si udivano come se fossero in chiesa. Compare Tino non aveva potuto durarla a campare d'aria sino a quel giorno, e aveva dovuto rivendere ogni cosa allo zio Crocifisso, per riavere i suoi denari. (G. Verga, I Malavoglia, p.162) $)^{31}$

B. O di un verbo transitivo a regime diretto sostantivo: 
- Ognuna diceva la sua dello zio Crocifisso, il quale piagnucolava sempre, e si lamentava come Cristo in mezzo ai ladroni, e intanto aveva denari a palate. (G. Verga, I Malavoglia, p.72) $)^{32}$

- A tratti lo prendeva il desiderio isterico di dire la verità, quella sua, la sola possibile, e andarsene; ma lo tratteneva un senso di compassione; e poi non era stato lui il primo a illudere Lisa con quel suo abbraccio? (A. Moravia, Gli Indifferenti, p.57)

C. Ma i casi più interessanti sono quelli in cui si ricorre a sintagmi vicari del verbum dicendi e la scelta dei quali sarà strettamente legata al registro linguistico di ogni autore:

- I vecchi in quel tempo si erano messi a discorrere fra di loro sotto il nespolo, colle comari che facevano cerchio e cantavano le lodi della ragazza, com'era brava massaia, che teneva quella casa meglio di uno specchio. (G. Verga, Malavoglia, p.145) ${ }^{33}$

- Donna Rosolina diede una buona lavata di capo a suo fratello, appena venne a sapere che si era messo in quel pasticcio, perché quelli della sciabola bisogna tenerseli amici. (G. Verga, Malavoglia, p.188) 34 $^{34}$

D. Talvolta il verbum dicendi anticipato è il verbo reggente di un DD che trova nel DIL la sua continuazione:

- "Vai" gli avrebbe detto; "Lisa ti aspetta". El'avrebbe gettato tra le braccia della donna; dove glieli avrebbe dati i denari Leo? Qui, nella casa di Lisa, sotto gli occhi della donna o altrove? Altrove. Poi discretamente, se ne sarebbe andato, chiudendo dietro di sé la porta, augurando la buona notte [...]; che notte senza fine! (A. Moravia, Gli Indifferenti, p.242)

E. Situazione analoga si verifica quando un verbum dicendi, inizialmente introduttore di un DI, perde progressivamente la sua capacità reggente per un indebolimento dei legami di subordinazione e «lascia così grammaticalmente libero il seguito del discorso» ${ }^{35}$ :

- Si ricordò che quel parente doveva venire nel mattino... e perché non prendere seriamente tutta la facenda e giovarsene? ... perché non continuare negli infingimenti? (A. Moravia, Gli Indifferenti, p.55)

- Dapprima credette di veder male, riguardò, sì, non c'era dubbio, era proprio l'amante, vestita di grigio col cappello in testa [...]. (A. Moravia, Gli Indifferenti, p.195)

Negli esempi sopracitati, il primo capoverso è narrazione oggettiva, il narratore analizza lo stato d'animo del personaggio; c'è però presenza implicita di questo, che sostituisce nel secondo capoverso l'analisi del narratore col proprio ragionamento in DIL. Dunque, dopo le frasi al DI seguono frasi di DIL nettamente individuabili; si 
osserva inoltre, che i verbi introduttivi sono stati omessi, e con essi anche la congiunzione che.

F. Ci sono poi casi in cui il verbum dicendi è intercalato nel brano enunciativo; in genere si tratta di verbi transitivi che non esercitano reggenza a causa della loro natura incidentale. Altra caratteristica di questi verbi di dire è il loro tempo, cioè l'imperfetto:

- $\quad[.$.$] si conoscevano ormai troppo bene per divenire marito e moglie... meglio$ andar via... separarsi... oppure, chissà? ... oppure restar così... amanti. Nel suo primo impulso di disgusto, nel suo primo istintivo movimento di difesa di quella pura e lontana idea del matrimonio, ogni più vile e penosa situazione le pareva preferibile alle nozze; pensava, ma non sapeva parlare, come affascinata dal sorriso e dagli sguardi dell'amante [...]. (A. Moravia, Gli Indifferenti, p.297)

G. Per finire, anche i verba dicendi posticipati al DIL possono essere transitivi, ma senza compiere azione subordinante:

- [...] come sempre, sarebbe ricaduto in quella mortale indifferenza che gli impediva di agire e di vivere come tutti gli uomini; abbastanza aveva giuocato con le sue fantasticherie; ora bisognava tentare di essere una buona volta tragico e sincero: "ora o mai" pensò. (A. Moravia, Gli Indifferenti, p.225)

I verbum dicendi posticipati al DIL possono essere, anche, sintagmi vicari (sostitutivo) del verbum dicendi:

- Quando uno non riesce ad acchiappare la fortuna è un minchione, questo si sa. Don Silvestro, lo zio Crocifisso, padron Cipolla, e massaro Filippo non erano minchioni, e tutti facevano loro festa, [...]. Piuttosto voleva starci lui, colle mani sulla pancia, la domenica e il lunedì, ed anche gli altri giorni giacché è inutile affaticarsi per nulla. Così 'Ntoni faceva il predicatore, come lo speziale. (G. Verga, I Malavoglia, p.224) ${ }^{36}$

oppure possono avvalersi di un deittico anaforico (come così) per attrarre nella loro sfera (senza subordinarlo) il brano enunciativo:

- Il mare russava in fondo alla stradicciola, adagio adagio, e a lunghi intervalli si udiva il rumore di qualche carro che passava nel buio, [...] e c'era pure della gente che andava pel mondo a quell'ora, e non sapeva nulla di compar Alfio, né della «Provvidenza» che era in mare né della festa dei Morti; - così pensava Mena sul ballatoio aspettando il nonno. (G. Verga, I Malavoglia, p.77) ${ }^{37}$

\subsubsection{I casi di un verbum dicendi assente}

Come abbiamo anticipato, ci sono casi in cui «il DIL è inserito ex abrupto nel contesto» per l'assenza del verbum dicendi, e questa è la condizione in cui si realizza al massimo la libertà del sintagma: 
- [...] ella riaprì gli occhi, si alzò a sedere, guardò: c'era Michele che la osservava con gli occhi scanzonati, come se al suo rimorso si fosse mescolato qualche altro sentimento; Carla che si sforzava di ricondurle le vesti sopra il petto scoperto; ma Leo? Dov'era Leo? Altrove che al suo fianco: aveva raccolto il portacenere e lo soppesava; poi bruscamente si volse verso Michele [...]. (A. Moravia, Gli Indifferenti, p.158)

- La sua fantasia illusa immaginava che Leo l'avrebbe presa tra le sue braccia, l'avrebbe scossa, chiamata per nome, si sarebbe alfine inquietato non vedendola rinvenire... e alfine ella sarebbe lentamente tornata in sé, avrebbe riaperto gli occhi, i primi sguardi sarebbero stati per l'amante, per lui il primo sorriso. Ma non fu così, Leo non la prese fra le sue braccia né la chiamò per nome. (A. Moravia, Gli Indifferenti, p.157).

Nel gruppo degli esempi citati, tratti da Gli Indifferenti, l'autore passa dalla narrazione al DIL, senza che segni ortografici e formule introduttive preparino tale passaggio, il quale più che vedersi si sente.

In simili circostanze (cioè ex abrupto), è frequente «la presenza di verbi descrittivi della gestualità del personaggio che parla e pensa $»^{38}$ :

- Ora sedeva nella poltrona con atteggiamento nobile ed elegante; teneva le gambe accavalciate, i pantaloni impeccabilmente rialzati su delle calze di lana, la testa pettinata e lustra un po' reclinata sulla spalla, verso la sigaretta che con languido gesto la mano stringeva tra due dita; sulla sua faccia morbida, rasata e ovale, riflessi d'ironia si alternavano a repentini rabbuiamenti, come l'ombra e la luce sul volto di una statua; fumava e pensava. (A. Moravia, Gli Indifferenti, p.112)

Il DIL, in questi esempi, si riconosce da tanti indizi: l'uso di: l'imperfetto (sedeva, teneva, ... ecc.), i pronomi trasferiti, e descrizioni molto precise che non possono essere fatte che dal protagonista stesso (atteggiamento nobile ed elegante, con languido gesto la mano stringeva tra due dita). 


\section{Conclusioni}

Studiando le funzioni del discorso indiretto libero, ho dedotto che il DIL nasca tradizionalmente dall'esigenza di avvicinare la lingua letteraria alla lingua viva. Infatti, il DIL ha permesso di dar voce alle classi popolari e contadine. Il DIL serve per esprimere i pensieri dei personaggi, così i pensieri del narratore e quelli dei protagonisti confluiscono, e diventa molto difficile distinguere tra narratore e personaggio; quindi serve per vedere cose e fatti con gli occhi e le parole dei personaggi. Esso consente di realizzare una forma di polifonia basata sulla simultaneità: non una voce, quella del narratore o quella del personaggio, bensì tutte e due le voci. Il DIL, inoltre, riflette i suoi effetti sulla ricezione del messaggio, inducendo una percezione diversa rispetto a quella del DD e del DI. Si usa, infine, come mezzo di distanziamento ironico dai personaggi o all'opposto di identificazione e di empatia con questi, veicolo del cosiddetto 'flusso di coscienza'.

Esaminando il DIL ne Gli Indifferenti sul livello morfo-sintattico, ho dedotto che il DIL ha come indicatori primari il sistema dei tempi verbali: il presente, il condizionale composto, l'imperfetto e l'infinito; e l'indipendenza del verbum dicendi: ho osservato la presenza e l'assenza del verbum dicendi: esistono tutte e due nello stile di Moravia nella stessa misura, ma il caso dell'assenza è più interessante in quanto rappresenta la massima libertà del sintagma e quindi più adatto al DIL. 


\section{Bibliografia}

\section{Libri:}

1. Calaresu, Emilia, Il discorso riportato, una prospettiva testuale. Modena, Edizioni il Fiorino, 2000.

2. Calaresu, Emilia. Testuali parole, la dimensione pragmatica e testuale del discorso riportato, Mlilano, FrancoAngeli, 2009.

3. Dardano M., Trifone P., Grammatica italiana con nozioni di linguistica, Brossura, 1995.

4. Mortara Garavelli, Bice, "il Discorso riportato", in: Lorenzo Renzi, Giampaolo Salvi, Anna Cardinaletti, Grande grammatica italiana di consultazione, Bologna, Il Mulino, 1995, 429-470.

5. Pasolini, Pier Paolo, "Intervento sul discorso libero indiretto", in: Paragone, rivista mensile di arte figurativa e letteratura, anno XVI, $\mathrm{n}$. 184/4, Firenze, Sansoni, pp. 121-144.

6. Cane, Eleonora. Il discorso indiretto libero nella narrativa italiana del Novecento. Roma, Silva Editore, 1969.

7. Dardano, Maurizio, Stili provvisori: la lingua narrativa italiana d'oggi, Roma, Carocci, 2010.

8. Faini Paola, "Il discorso indiretto libero nel testo narrativo", in: Quaderno del Dipartimento di letterature comparate 2, Dipartimento di Letterature Comparate -Università degli Studi Roma Tre, Carocci, Roma, 2006, pp. 3546.

9. Herczeg, Giulio, Lo stile indiretto libero in italiano, Firenze, Sansoni, 1963.

10. Mortara Garavelli, Bice, La parola d'altri-prospettive di analisi del discorso. Palermo, Sellerio, 1985.

11. Mortara Garavelli, Bice, "Il discorso indiretto libero in dissoluzione?", in: Linguistica e filologia - omaggio a Benvenuto Terracini, (a cura di) Cesare Segre, Milano, Il Saggiatore di A. Mondadori Editore,1968.

12. Mortara Garavelli, Bice, "il Discorso riportato", in: Lorenzo Renzi, Giampaolo Salvi, Anna Cardinaletti, Grande grammatica italiana di consultazione, Bologna, Il Mulino, 1995, 429-470.

13. Schettino, Franca, "Oggettività e presenza del narratore ne Gli Indifferenti di Moravia", in: Revue des études italiennes, XX/ n. 3-4, Paris, Librairie Marcel Didier, 1974.

14. Bach, Svend, 1972, "Lo stile indiretto libero nel Piacere di Gabriele D'Annunzio", in Studi di Grammatica Italiana, II, 1972, pp. 155-189. 
15. Bazzanella, Carla, «I modi dell' imperfetto», in: italiano e oltre, Rivista bimestrale, A. 1, n. 1, Firenze, La Nuova Italia, 2002: pp.18-21.

16. Serianni, Luca, Italiano in prosa, Firenze, Franco Cesati Editore, 2012.

17. Bendoni, Anna Danesi, "Grammaticalizzazione del discorso indiretto libero nei «Malavoglia»", in: Studi di grammatica italiana, a cura di Accademia della Crusca, Vol. IX, Firenze, 1980: 253- 271.

\section{Opere:}

1. Giovanni Verga, I Malavoglia, Milano, Treves, 1881.

2. Bruno Cicognani, La Velia, Firenze, Mauro Pagliai, 1966.

3. G. Verga, Mastro-don Gesualdo, in Opere, Milano-Napoli, 1995.

\section{Footnotes}

${ }^{1}$ Emilia Calaresu, Il discorso riportato, una prospettiva testuale. Modena, Edizioni il Fiorino, 2000, 27.

2 ibidem.

${ }^{3}$ Maurizio Dardano, Pietro Trifone, Grammatica italiana con nozioni di linguistica, Brossura, 1995, 751.

${ }^{4}$ Emilia Calaresu. Testuali parole, la dimensione pragmatica e testuale del discorso riportato, Mlilano, FrancoAngeli, 2009, 29.

${ }^{5}$ ibidem.

${ }^{6}$ Mortara Garavelli, Bice, "il Discorso riportato", in: Lorenzo Renzi, Giampaolo Salvi, Anna Cardinaletti, Grande grammatica italiana di consultazione, Bologna, Il Mulino, 1995, 464.

${ }^{7}$ Cfr. Eleonora Cane, Il discorso indiretto libero nella narrativa italiana del Novecento. Roma, Silva Editore, 1969, 43; Maurizio Dardano, Stili provvisori: la lingua narrativa italiana d'oggi, Roma, Carocci, 2010, 144.

${ }^{8}$ E. Cane, Il discorso indiretto libero nella narrativa italiana del Novecento, op. cit., p. 20.

${ }^{9}$ Paola Faini, "Il discorso indiretto libero nel testo narrativo", in: Quaderno del Dipartimento di letterature comparate 2, Dipartimento di Letterature Comparate -Università degli Studi Roma Tre, Carocci, Roma, 2006, p.37 .

${ }^{10}$ Bice Mortara Garavelli, La parola d'altri-prospettive di analisi del discorso. Palermo, Sellerio, 1985, 105.

${ }^{11}$ B. Mortara Garavelli, La parola d'altri-prospettive di analisi del discorso op. cit., p. 105.

${ }^{12}$ B. Mortara Garavelli, La parola d'altri-prospettive di analisi del discorso, op. cit., p.105. (17)

${ }^{13}$ E. Cane, op. cit., p.34.

${ }^{14}$ G. Herczeg, op. cit., p.76. 
15 ivi., p.78.

${ }^{16}$ Ogni elemento cui si riferisce nei commenti degli esempi citati, si scrive in grassetto.

${ }^{17}$ G. Herczeg, op. cit., p.81.

${ }^{18}$ Franca Schettino, "Oggettività e presenza del narratore ne Gli Indifferenti di Moravia", in: Revue des études italiennes, XX/ n. 3-4, Paris, Librairie Marcel Didier, 1974, p.306.

${ }^{19}$ Ibidem.

${ }^{20}$ Bisogna dire, però, che le esitazioni fra due cifre sono frequenti anche nel testo del narratore quando egli vuole evitare un punto di vista troppo superiore, cercando invece di mettersi nei panni dei personaggi. (cfr. Svend Bach, "Lo stile indiretto libero nel Piacere di Gabriele D'Annunzio", in Studi di grammatica italiana, (a cura dell'Accademia della Crusca), Vol. II, Firenze, G. C. Sansoni, 1973, p.168).

${ }^{21}$ P.P. Pasolini, op. cit., p.122.

${ }^{22}$ Ibidem.

${ }^{23}$ G. Herczeg, op. cit., p.64.

${ }^{24}$ B. Moratara Garavelli, "Il discorso indiretto libero in dissoluzione?", op. cit., p.36

${ }^{25}$ Carla Bazzanella, «I modi dell'imperfetto», in: Italiano e oltre, Rivista bimestrale, A. 1, n. 1, Firenze, La Nuova Italia, 2002. p.18.

${ }^{26}$ S'intende per il fenomeno d'immedesimazione il caso in cui diventa quanto mai incerto stabilire se è veramente l'autore che parla, come lascerebbe supporre la costruzione della frase, o i personaggi stessi che attraverso l'autore ci fanno giungere l'eco dei loro discorsi. Il fenomeno non è occasionale ma rappresenta un carattere fondamentale nell'opera di un autore costantemente teso alla percezione delle vibrazioni psicologiche più sottili ed a una rappresentazione del personaggio che si sviluppa partendo dall'interno. Su questo punto, Cfr. E. Cane, op.cit., p.26.

${ }^{27}$ Cfr. Massimo Palermo, Linguistica testuale dell'italiano, Bologna, Il Mulino, 2013, p.129.

${ }^{28}$ G. Herczeg, op. cit., p.45.

${ }^{29}$ Ibidem.

${ }^{30}$ Luca Serianni, Italiano in prosa, Firenze, Franco Cesati, 2012, p.119.

31 Anna Danesi Bendoni, "Grammaticalizzazione del discorso indiretto libero nei «Malavoglia»", in: Studi di grammatica italiana, a cura di Accademia della Crusca, Vol. IX, Firenze, 1980, p.257.

${ }^{32}$ Ivi., p. 258.

${ }^{33}$ Ivi., p. 257.

${ }^{34}$ Ivi., p. 258.

${ }^{35}$ Ibidem.

${ }^{36}$ Ivi., p. 260.

${ }^{37}$ Ibidem.

${ }^{38}$ Ibidem. 


\title{
الوظائف والخصائص النحوية_الصرفية للحديث المباشر غير الحر في اللغة الإيطالية
}

\author{
حنناء محمود أحمد محمود \\ مدرس مساعد بقسم اللغة الإيطالية \\ كلية الألسن - جامعة المنيا
}

منهج البحث

يتم معالجة موضوع البحث على المستويين النظري و التطبيقي، ويتم التطبيق على رواية "الكلامبالون" للكاتب الإيطالي ألبرتو مور افيا.

$$
\text { إثكالية البحث }
$$

ما هي الوظائف و الخصائص النحوية_الصرفية للحديث المبانشر غير الحر؟

ملخص البحث

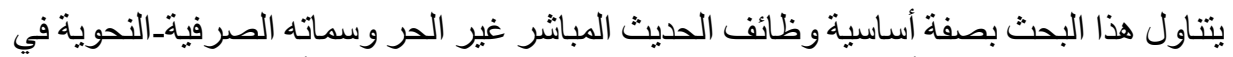
اللغة الإيطالية. في بداية البحث أقدم تعريفا لهذا التركيب: يعرف بشكل عام على الثئ أنه خليط ما بين الحديث المبانشر و الحديت غير المبانر.

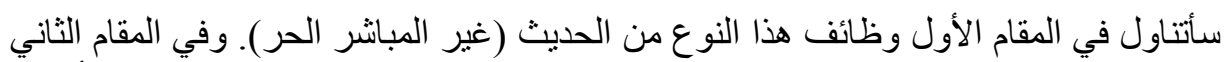

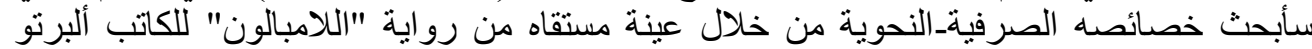
مور افيا. 